\title{
¿MUY JÓVENES PARA DECIDIR SU FUTURO? CAPACIDAD DE AGENCIA Y MOVILIDAD SOCIAL EDUCATIVA EN JÓVENES PROFESIONALES DE LA UNIVERSIDAD DE ANTIOQUIA ${ }^{1}$
}

\author{
VÍCTOR GUERRA ZABALA ${ }^{2}$ \\ CARLOS DUQUE MEJÍA ${ }^{3}$ \\ EDINSON BRAND MONSALVE ${ }^{4}$
}

\begin{abstract}
RESUMEN
Se presenta un análisis de las motivaciones que configuraron procesos de movilidad social educativa en un grupo de ochenta jóvenes egresados de la Universidad de Antioquia, para lo cual se aplicó una encuesta sobre el acceso, transcurso y egreso de la universidad. La conclusión principal es que la edad no es impedimento para construir el proyecto de vida y generar acciones que lleven a los jóvenes conscientemente a cumplir sus deseos a través de la educación universitaria, que les brinda satisfacción en el ser principalmente, evidenciando sus impactos positivos a pesar de las dificultades para acceder a ella.
\end{abstract}

PALABRAS CLAVE: EDUCACIÓN SUPERIOR, JÓVENES, CAPACIDAD DE AGENCIA.

\footnotetext{
${ }^{1}$ Artículo resultado del proyecto de investigación «Medición de impacto de la educación superior regionalizada de la Universidad de Antioquia. Estudio de caso de los programas de Ingeniería, Sociología y Educación en la sede del Carmen». Financiado por Vicerrectoría de Investigación de la Universidad de Antioquia, Comité para el Desarrollo de la Investigación CODI, a través de la «Convocatoria Programática 2016 Área de Ciencias Sociales, Humanidades y Artes».

${ }^{2}$ Sociólogo egresado de la Universidad de Antioquia, estudiante de la Maestría en Desarrollo Regional del Colegio de la Frontera Norte (COLEF), participante del Grupo de investigación: Redes y Actores Sociales Universidad de Antioquia. Correo electrónico: vguerra.mdr2020@colef.mx

3 Sociólogo egresado de la Universidad de Antioquía, participante del Grupo de investigación: Redes y Actores Sociales Universidad de Antioquia. Correo electrónico: carturo.duque@udea.edu.co

${ }^{4}$ Candidato a doctor en Filosofía, docente de la Universidad de Antioquia, participante del Grupo de investigación: Redes y Actores Sociales Universidad de Antioquia. Correo electrónico: edinson.brand@udea.edu.co
} 


\title{
JOVENS DEMAIS PARA DECIDIR SEU FUTURO? \\ CAPACIDADE DE AGÊNCIA E MOBILIDADE SOCIAL EDUCATIVA EM JOVENS PROFISSIONAIS DA UNIVERSIDADE DE ANTIOQUIA
}

\begin{abstract}
RESUMO
É apresentada uma análise das motivações que configuraram processos de mobilidade social educativa em um grupo de oitenta jovens formados da Universidade de Antioquia, para o qual foi aplicada uma pesquisa sobre o acesso, anos de estudo e formatura da universidade. A principal conclusão é que a idade não é impedimento para construir o projeto de vida e gerar ações que levem os jovens conscientemente a realizar seus desejos por meio da educação universitária, que entrega satisfação no ser principalmente, evidenciando seus impactos positivos apesar das dificuldades para aceder a ela.
\end{abstract}

PALAVRAS-CHAVE: ENSINO SUPERIOR, JOVENS, CAPACIDADE DE AGÊNCIA.

\section{ARE THEY TOO YOUNG TO DECIDE ON THEIR FUTURE? AGENCY CAPACITY AND EDUCATIONAL SOCIAL MOBILITY IN YOUNG PROFESSIONAL PEOPLE FROM UNIVERSIDAD DE ANTIOQUIA}

\begin{abstract}
The present work brings up an analysis on the motivations behind the educational social mobility processes in a group of eighty young graduates from Universidad de Antioquia, based on a survey about university access, study years and graduation. It is mainly concluded that age is not a hindrance to either build a life project or to generate actions from which young people consciously fulfill their desires through university education; instead, higher education gives them satisfaction, evidencing its positive impact on them in spite of the difficulties to access to it.
\end{abstract}

KEYWORDS: HIGHER EDUCATION, YOUNG PEOPLE, AGENCY CAPACITY. 


\section{INTRODUCCIÓN}

La modernidad tardía está llena de liturgias, exigencias, razonamientos y rutinas que estructuran las acciones y motivaciones de los agentes humanos, a la vez que estos producen nuevas e innovadoras formas de comunicación y organización social (Giddens, 2011, 1997, 2018). En la actualidad, es bastante problemático, y casi imperceptible, el grado en que se desestiman y son reemplazadas las actividades sociales tradicionales y su impacto sobre las prácticas futuras de los seres humanos. Para Giddens, Bauman, Bourdieu y Beck, la sociedad capitalista actual se mueve más «rápido» que otras formas de totalidad societal (Chávarro, 2018; Giddens, 1997). Indudablemente, el sistema capitalista posee una base económica de competencia a escala global, sus efectos son ya conocidos en el comercio intersectorial, los mercados segmentados y oligopolistas. Lo revela la inmensa preocupación y angustia que viven los agentes cuando se trata de asegurar, socioeconómicamente, el futuro (Giddens, 1997). Uno de los niveles más importantes de este problema recae en el cuerpo, el cual, al igual que el sujeto epistémico, pasa a ser un terreno de interacción social sostenida, de peticiones, exigencias y expectativas que se ordenan sistemáticamente en relación con la demanda del otro(s) colectivo (Giddens, 1997: 275). Según relata Corominas (2016), «el cuerpo está muy influido por nuestras experiencias sociales y por las normas y valores de los grupos a los que pertenecemos» (p. 121).

El cuerpo es una construcción social dinámica, posee varias etapas cronológicas, ontológicas (identitarias), fisiológicas, anatómicas, culturales y psicológicas. Etapas que se legitiman constantemente en la interacción social cotidiana con otros seres humanos (Giddens, 2000, 1997, 2011). La juventud es una de estas etapas culturales donde estas demandas y expectativas se agudizan o aumentan en su magnitud. Sin embargo, no se trata de transformaciones y alegatos simples en la externalidad del individuo; según Giddens (2011), estas demandas, las cuales conciernen a las reglas de la modernidad, alteran de manera radical la naturaleza de la vida social cotidiana y afectan a los aspectos más personales e íntimos de la experiencia inmediata (Giddens, 1997, 2011). La educación profesional es una de tantas demandas que por lo general se desarrolla intensamente en esta etapa de vida, sin mencionar que los procesos de identificación grupal cumplen un rol cada vez mayor en los intereses, motivaciones y prácticas sociales de los jóvenes. En consecuencia, los grupos sociales, según lo relatan varios autores como Goldthorpe y Erikson, «[...] 
intervienen en las influencias sociales, cuyas consecuencias e implicaciones son de carácter universal, y las fomentan de manera directa» (Giddens, 1997: 10). Es evidente que los jóvenes, como grupo social, juegan un papel determinante en los cambios sociales que surgen entre generaciones. Este grupo social se encuentra en una posición tercia o media entre la reproducción de las costumbres tradicionales de sus predecesores y la producción reflexiva de nuevas experiencias para sus sucesores.

Los jóvenes han sido objeto de estudio de diversas disciplinas científicas y humanas (Izquierdo Cázares y Mestanza Páez, 2017). En la bibliografía especializada existen diferentes formas de comprender el espíritu de dicho objeto. Cuando se utiliza el término jóvenes se hace referencia a la naturaleza objetiva y universal de la edad, esto es, la experiencia de las poblaciones en sus diferentes vicisitudes temporales (Filardo, 2018). Esta edad cronológica posee ciertos preceptos, mandatos y códigos; la juventud se refiere a estos imperativos culturales que dominan o gobiernan cierto rango de edad (Izquierdo Cázares y Mestanza Páez, 2017). Pero la edad de los jóvenes, aunque se presenta como evidente, «es producto de una construcción sociocultural, y por tanto siempre es situada (en un espacio-tiempo y posición social dada [...]» (Filardo, 2018: 11). Ambos conceptos son representados en el discurrir ordinario de la vida social con un sentido peyorativo y muchas veces asociado a la falta de voluntad. Esto se debe, en parte, al carácter de concebirlos como una etapa cronológica y cultural con poca cautela, donde las motivaciones son casi instintivas y la experiencia poco razonable y práctica. Este proceso de construcción social adolece de vicios instaurados en el orden capitalista reciente, donde se generan exigencias educativas, sociales, laborales y estéticas cada vez mayores desde la óptica de los adultos. Los jóvenes no solo se enfrentan a un mundo glocalizado que es cada vez más exigente y complejo, también lo hacen bajo condiciones de estigmatización, exclusión y riesgo social.

El mayor interés de la literatura especializada sobre jóvenes y educación se ha enfocado más en las problemáticas sobre el acceso a la educación y sobre la formación profesional (Angelucci et al., 2017: 540). La normalización de la precariedad entre las personas jóvenes en la literatura es amplia; aunque es cierto que los aspectos psicosociodemográficos son relevantes en esta etapa de vida (Córdova Osnaya et al., 2007), el análisis de la situación documental demuestra que sobre los jóvenes recae una imagen negativamente sesgada, asociada a la violencia, el consumo de drogas en las universidades, hábitos inadecuados de alimentación, falta de oportunidades de adquisición de capital, problemas de 
ansiedad y depresión (Córdova Osnaya et al., 2007). A los jóvenes «suele atribuírsele, en su búsqueda y en su rebeldía, lo más sublime o lo más peligroso y destructivo» (Cohen, 2017). En las mujeres la situación de estigmatización se agudiza mucho más, y son pocos los estudios que utilizan un enfoque sobre desigualdad de género para estudiar la relación entre la edad y la educación superior. En este sentido, los jóvenes son relegados de las decisiones políticas importantes, la producción de su trabajo les es enajenada en los grupos familiares y de pertenencia, lo cual implica que son sujetos marginados a posiciones de dependencia subjetiva y en otros sistemas institucionales les son impuestos saberes acríticos a través de la transmisión intergeneracional de conocimiento (Westendarp Palacios e Iglesia Sahagún, 2019: 8).

Todas estas visiones estáticas han derivado en la generación y consolidación de un discurso sobre la existencia de una baja capacidad de agencia en esta población, en razón de lo cual el propósito de esta investigación se orientó a comprender la experiencia de movilidad social educativa de ochenta jóvenes egresados de la Universidad de Antioquia; problematizando la experiencia de los sujetos a la luz del concepto de capacidad de agencia propuesto por Anthony Giddens. Este concepto destaca, entre otras cosas, la importancia de las motivaciones de las personas para construir, reflexionar y emprender acciones como agentes de cambio. Las personas saben mucho sobre las condiciones y consecuencias de lo que hacen en su vida cotidiana, por ende, todos los seres tienen una voluntad para «salir adelante» (Bialakowsky, 2018), bajo motivaciones, decisiones, intereses y expectativas que pueden variar en cualidades. Los principales resultados sugieren la existencia de un proceso de movilidad social educativa de jóvenes profesionales. Este cambio es producto, en parte, de las motivaciones e intereses, que son adquiridos y desarrollados por estos jóvenes para generar ciertos cambios y logros en su trayectoria particular de vida futura.

\section{ESTADO DEL ARTE}

La educación superior es percibida como un factor de oportunidad de vida para los jóvenes (Tarabini y Curran, 2015; Vega et al., 2017). Para la teoría del capital humano, los jóvenes que estudian son inversores racionales «homoeconomicus»: invierten en su capital intelectual como parte de un costo inicial para incrementar la magnitud de sus riquezas o rentas (beneficio agregado) 
(Giddens, 2000). Desde esta línea, la movilidad social educativa se observa como un proceso que depende directamente del esfuerzo individual, es decir, «para surgir en la vida hay que poner mucho esfuerzo, estudiar mucho, trabajar bien» (Denegri et al., 2009). La acción individual pasa así a estar orientada por la lógica de la inversión y el retorno, en la que se analizan variables como el salario y el tipo de contratos laborales. En efecto, «la educación superior es [...] uno de los principales vehículos legítimos de movilidad social ascendente y, por lo tanto, un medio para lograr a largo plazo mayor equidad social» (Moo Estrella y López Santillán, 2019: 11). Para estos estudios, en la actualidad, ya no basta con la búsqueda «activa» de empleo por parte de los jóvenes, ahora hay que «invertir» en la construcción lógica del ser (Santos Ortega y Muñoz Rodríguez, 2017: 137). La mayoría de las investigaciones concluyen que la educación es el factor que más incide sobre el estatus de estos jóvenes en el mercado de trabajo. Esta línea, por lo general economicista, suele descuidar los aspectos asociados al origen social de los familiares y grupos cercanos (Yaschine, 2015).

Otros estudios prefieren destacar que los jóvenes enfrentan desafíos complejos, pero algunos de ellos lo hacen con menores recursos y en contextos de oportunidades de mayor precariedad (Yaschine, 2015: 400). Aunque son muy variadas las formas de comprender el fenómeno, esta línea, que puede denominarse estudios sobre la desigualdad, enfatiza las condiciones estructurales de los jóvenes. Según Jaramillo et al. (2012), en los jóvenes se hace más evidente el peso del origen familiar, ya que son estos el sector de la población que más compite por las oportunidades educativas, y de esta manera lo hacen por las laborales. En esta línea, muchas investigaciones prefieren estudiar este campo en relación con el origen social de los jóvenes, ya que este influye de manera considerable en la construcción de un tipo particular de exigencias asociadas a la juventud. Sostienen, además, que la influencia se expresa de distintas maneras: física, verbal o simbólica (Arias Cardona y Arias Gómez, 2018). El paso por la universidad no es fácil y suele representar ciertas complejidades asociadas a la diferencia (Arias Cardona y Soto, 2019), en donde el efecto de la clase social sobre las oportunidades, creencias y deseos educativos de los jóvenes es significativo y muchas veces inhabilitante. Según relata Giddens (1979), la clase social sigue siendo una variable crucial en los análisis de movilidad social; para el autor, las posibilidades de ascender en la estructura tienden a permanecer cerradas para los grupos con menos poder económico y social, sobre todo en los más altos niveles del logro académico. 
Tanto los países desarrollados como muchos en proceso de desarrollo experimentaron un proceso de expansión educativa (Goldthorpe y Breen, 2010 citado en García Correa, 2017). Cartagena (2005) explica que Colombia, al igual que la mayoría de los países después del período de posguerra, contó con una importante expansión educativa (total bruto de número de matriculados y número de instituciones creadas), que trajo como resultado un aumento de los años promedios de educación entre las diferentes cohortes. Sin embargo, muchos estudios coinciden en que a mediados de los años setenta, a nivel nacional, se presentó un descenso en el ritmo de crecimiento de la movilidad social educativa ascendente (García Correa, 2017). Por otra parte, se dice que el mercado de trabajo formal en Colombia ha adquirido mayor fluidez y dinamismo después del último trimestre de 2010, coincidiendo con una caída sustancial de la tasa de desempleo (Cartagena, 2005). Sin embargo, la población juvenil comprendida entre los 18 y los 25 años aproximadamente es uno de los grupos de la población más golpeado por la desocupación (García Correa, 2017; Cartagena, 2005), lo que se debe, en parte, a la hostilidad del mercado laboral con los jóvenes recién graduados. A la falta de oportunidades de trabajo, por la escasa generación de empleo y la ineficacia de la política institucional, se le ha aunado la falta de cobertura educativa para muchos sectores poblacionales y las altas exigencias de experiencia laboral y social que demandan los empleadores sobre los jóvenes (Blanco Rivera, 2007).

Finalmente, existen otras líneas de estudios, donde se problematiza el rol de las redes familiares (Vega et al., 2017: 185), redes virtuales entre jóvenes (Westendarp Palacios e Iglesia Sahagún, 2019) y las trayectorias sociales como posible enfoque que permita analizar las juventudes (Dávila León y Ghiardo Soto, 2018).

\section{CAPACIDAd DE AGENCIA}

En el ámbito académico, aún existe una postura general objetivista sobre la movilidad social (Giddens, 2000). Muchas de estas se desprenden del cuestionamiento sistemático a la categoría del sujeto de la filosofía de René Descartes, y adoptan, de forma axiomática, la teoría de la regulación causal de Hume (Castillo Murillo, 2019). Esta postura, a la que Giddens denomina abordaje estructuralista, considera que los seres humanos son seres inertes carentes de capacidad de acción, pues sus motivaciones, intereses y sentidos son 
profundamente subjetivos y ambiguos (Giddens, 2018: 217). De forma contraria, para Giddens, «todos los seres humanos son agentes entendidos. Esto significa que todos los actores sociales saben mucho sobre las condiciones y consecuencias de lo que hacen en su vida cotidiana» (Giddens, 2011: 307). Ser un agente significa ser un individuo con la capacidad de hacer una diferencia, de intervenir en el curso habitual o «normal» de los acontecimientos del mundo (Pavez Soto y Sepúlveda Kattan, 2019). De manera que, un agente posee un entendimiento sobre las actividades cotidianas, sociales y culturales en las que participa, incluidas aquellas que no caen directamente dentro del entorno perceptivo del individuo (Giddens, 1997, 2011; Cruz Santana, 2017). Desde este enfoque, los seres humanos controlan y desarrollan acciones sociales, las cuales tienen consecuencias muy concretas (Aranda Reyes, 2016: 14; Bialakowsky, 2018). En otros términos, «si se le pregunta sobre ello, los agentes son normalmente capaces de dar interpretaciones discursivas de la naturaleza del comportamiento adoptado y de las razones de este» (Giddens, 1997: 51).

El historiador David Gary Shaw (2001) ha buscado en el estructuracionismo del sociólogo inglés un antídoto contra los excesos del antihumanismo estructuralista (citado en Castillo Murillo, 2019: 217), incorporando una mirada según la cual las personas, en este caso los jóvenes, poseen un alto entendimiento y participación en actividades sociales cotidianas que tienen una fuerte influencia en un conjunto de acciones y valores agregados, tales son, el logro educativo, laboral, profesional y personal de los sujetos implicados. Según Giddens, debe ponerse el foco de atención en los elementos activos de la agencia (Bialakowsky, 2017). Así, por un lado, Giddens (2012) plantea que la agencia, en su discurrir diario, implica una decisiva capacidad de transformación de los sujetos sobre su vida misma, lo que los lleva a racionalizar ciertas actividades y objetivos, reflexionar sobre las oportunidades que poseen para lograr dicho valor e impulsar acciones bajo la forma de una motivación extraordinaria (Bialakowsky, 2018; Pavez Soto y Sepúlveda Kattan, 2019). Cada uno de estos aspectos del agente social tiene una consecuencia en el futuro de los individuos, de sus grupos cercanos y de la sociedad que habita.

Similar al concepto de motivación social planteado por Weber, para la teoría de la estructuración, este denota el potencial de una acción, generalmente, unos motivos proveen planes generales dentro de los que se escenifica una conducta humana (Giddens, 2011). Buena parte de nuestras acciones cotidianas no reconoce motivación directa, pues se trata del impulso inconsciente de la acción. Aunque existen ciertas reservas sobre la interpretación freudiana de la 
naturaleza de lo inconsciente, para Giddens (2011), la motivación de la acción es una característica importante de la capacidad de agencia; ella ayuda a configurar ciertos proyectos, planes y modos de vida. Los motivos están intrínsecamente relacionados con los intereses, las expectativas y las actividades sociales que desarrollan los agentes sociales. En consecuencia, las decisiones que toman los sujetos de ordinario, que pueden ser tan simples como tomar el transporte local que se dirige hasta el establecimiento educativo, o tan complejas que orientan los valores como un contrato matrimonial, influyen en la estructura social de forma retroactiva en el propio agente, como una dualidad de estructura (Giddens, 2000, 2011). «Lo que nos hace ver Giddens es que, tras las actividades cotidianas, aparentemente realizadas sin esfuerzo y sin problemas, se lleva a cabo un constante trabajo de mantenimiento y reproducción social» (Corominas, 2016: 123).

En relación con la capacidad de agencia y la movilidad social, ambos fenómenos poseen un grado de interacción, así lo ilustra Giddens en el apartado final de su obra La constitución de la sociedad: bases para la teoría de la estructuración (2011): la movilidad está relacionada estrechamente con la conducta y las prácticas de un individuo. De tal forma que los sujetos poseen un entendimiento «estratégico» o táctico sobre las relaciones e intereses que se establecen en la sociedad (Bialakowsky, 2017), y tales intereses son impulsados y desarrollados por determinadas motivaciones sociales. En consecuencia, por movilidad social se puede comprender el movimiento o desplazamiento de agentes sociales entre distintas posiciones socioeconómicas al interior de una estructura, que puede ser descrita en términos de grupos, clases, estamentos, estratos, círculos, logros (Giddens, 1992; Kerbo, 2003). Esta estructura puede definirse como «las desigualdades estructuradas entre diferentes agrupamientos de individuos [...] mientras que la movilidad es lo que ocurre con los individuos que la desempeñan» (Giddens, 1992: 237). Existen varios tipos de movimiento, como lo es la movilidad educativa intergeneracional, la cual hace alusión a la posibilidad de cambiar un nivel educativo respecto al que tuvieron sus padres o grupos de pertenencia (García Correa, 2017). Por ende, la movilidad social puede estudiarse comprendiendo la motivación de un grupo de individuos que buscan modificar esas desigualdades estructuradas con respecto a los procesos de reproducción (Timasheff, 1961). 


\section{MÉtodo}

La metodología empleada en esta investigación fue cuantitativa no experimental basada en un estudio de tipo estadístico descriptivo, que se caracteriza por medir las magnitudes de los fenómenos sociales o problemas de investigación estudiados (Hernández Sampieri et al., 2014: 5-6). Igualmente, la estadística descriptiva permite comprender bajo ciertas propiedades numéricas algunas dimensiones de los hechos sociales, como los atributos poblacionales relacionados con variables de corte demográfica, cultural y socioeconómica (Bar, 2010: 294-295; Hernández Sampieri et al., 2014). Este nivel se abordó por ser considerado suficiente para el análisis de ciertos procesos sociales para los cuales es necesario explicar y describir el comportamiento de un grupo de individuos; además, la estadística descriptiva posibilita obtener información sobre con qué frecuencia se presenta la variable de estudio, de qué forma se distribuye, si los datos están dispersos o si se concentran en un punto central, entre otros aspectos (Camacho, 2003, citado en Espinosa Garzón, 2017: 10). Para el análisis de la información se hizo uso de la distribución de frecuencias bivariada $y$ multivariada, medidas de tendencia central y dispersión. Debido a no se cuenta con criterios de inclusión poblacional, diferentes a ser jóvenes, egresados y egresadas de la institución educativa estudiada y a falta de información empírica recolectada hasta el momento, fue necesario aplicar un muestreo representativo a conveniencia, puesto que se seleccionaron aquellos casos accesibles que cumplían con los objetivos trazados (Otzen y Manterola, 2017).

En total, se encuestaron ochenta jóvenes del seccional oriente de la Universidad de Antioquia, que se encuentra ubicada en una de las nueve subregiones del departamento de Antioquia en Colombia, con sede específicamente en el municipio del Carmen de Viboral, ubicado a $44 \mathrm{~km}$ aproximadamente de la ciudad de Medellín. De forma global, la investigación abordó una población de profesionales de Sociología 18,8\%, Psicología 18,8\%, Educación 37,5\%, Ingeniería 22,5\% y Biología 2,5\%. Todos ellos se encuentran entre las edades de 14-28 años. El referente para la delimitación de la población en función de la edad se construyó con base al marco legal colombiano bajo la Ley 1622 de 2013 «estatuto de ciudadanía juvenil», la cual considera que jóvenes son todas aquellas personas entre 14 y 28 años cumplidos. Resulta idónea tal normativa porque establece el marco institucional en el que se respalda el ejercicio de derechos ciudadanos juveniles, relacionados con el sistema educativo, las políticas de empleabilidad juvenil, los voluntariados y la elección de consejo juvenil, entre otras políticas públicas. Cabe agregar que frente a las 
variables sexo, género, etnia y pertenencia territorial (urbano/rural), no se observaron, en el nivel descriptivo, diferencias significativas en relación con el objeto de estudio.

El instrumento que se utilizó corresponde a un cuestionario compuesto de 84 ítems, y se aplicó de forma presencial, con una duración promedio de 45 minutos en cada caso. Estos ítems dan cuenta de tres ejes temáticos: el primero hace referencia a la situación y disposición económica, educativa y personal de familiares y egresados antes de ingresar a la universidad, nivel educativo más alto obtenido, expectativas de acceso a la educación superior, prácticas intelectuales cotidianas, grupo de personas que influyeron en la decisión de acceder a la universidad; el segundo, al proceso formativo en la universidad, características relevantes del rendimiento académico, apoyos recibidos por parte de otras instituciones, participación en actividades extracurriculares y oficiales; el tercer y último momento se preguntó por la situación actual de los jóvenes, ejercicio de la profesión, ingresos salariales, oferta de empleos y percepciones sobre éxito profesional, personal, económico y laboral.

\section{Resultados}

\subsection{Motivación EdUCATIVA}

Los avances en la formación educativa son uno de los factores más relevantes de la movilidad educativa intergeneracional. La educación está relacionada con la capacidad que tienen los sujetos de obtener una ganancia cognitiva en un determinado plazo de tiempo; entre más rápido e inmediato sea el logro y la obtención de credenciales especializadas, mayores oportunidades pueden desprenderse de la continuidad educativa. En este sentido, la educación es una inversión de tiempo y esfuerzo, que demanda una serie de habilidades e intereses dispuestos en el cuerpo y la mente de las personas. En el estudio realizado se observa una evolución constante de la trayectoria educativa de los jóvenes. En promedio, la edad a la que ingresan estos jóvenes a la universidad es semejante a la edad promedio a nivel nacional en la que los jóvenes terminan su educación media escolar. Según lo relatan distintos medios de comunicación, el $40 \%$ de los jóvenes colombianos no termina la educación media, y los que logran concluir en condiciones de normalidad académica lo hacen a los 17 años aproximadamente (Pérez Martínez, 2017). De hecho, se observa que la mayoría 
de los jóvenes que ingresaron a la universidad lo hicieron a los 17 años, con una media de 17,2 y una desviación estándar de 1,1, la edad mínima registrada fue 15 y la máxima de 21 años. Esto significa que se trata de un grupo de jóvenes bastante homogéneo, con una continuidad educativa entre la educación media y la educación superior o universitaria, lo que ha acotado el trecho temporal entre la escuela y el acceso a la universidad.

La trayectoria escolar de estos jóvenes frente a sus estructuras de parentesco los hace pioneros en la formación superior, esto debido a que lograron superar de forma significativa el lugar más alto alcanzado por sus abuelos, madres y padres, en lo concerniente a la obtención de conocimientos, habilidades y credenciales. Al respecto, tal como lo indica el Gráfico 1, el 31,3\% de los abuelos no obtuvo ningún tipo de formación escolar, comportamiento similar para aquellos que culminaron en el grado de primaria o secundaria; 1,3\% logró un título de educación técnica o tecnológica, tan solo el 2,5\% culminó un título de educación profesional o de pregrado, y ninguno de ellos logró la educación posgraduada. Frente al padre, el $67,5 \%$ de ellos terminaron la educación primaria o secundaria, comportamiento que en las madres es semejante entre los primeros niveles educativos, pero diferente entre los últimos, el 82,6\% logró terminar la primaria o la secundaria. En el caso de los padres existe una disminución en relación con la educación profesional de los abuelos, pero un aumento en la educación posgraduada, que para el caso de las madres es de 2,5\% y de los padres de $1,3 \%$. Frente a los hijos graduados, es de aclarar que el 100\% de ellos tiene un título de pregrado y el $23,8 \%$ se encuentra a punto de terminar o había terminado un proceso de formación posgraduada. 
GRÁFICO 1. TÍTULOS EDUCATIVOS MÁS ALTOS OBTENIDOS POR GRUPO DE PERSONAS

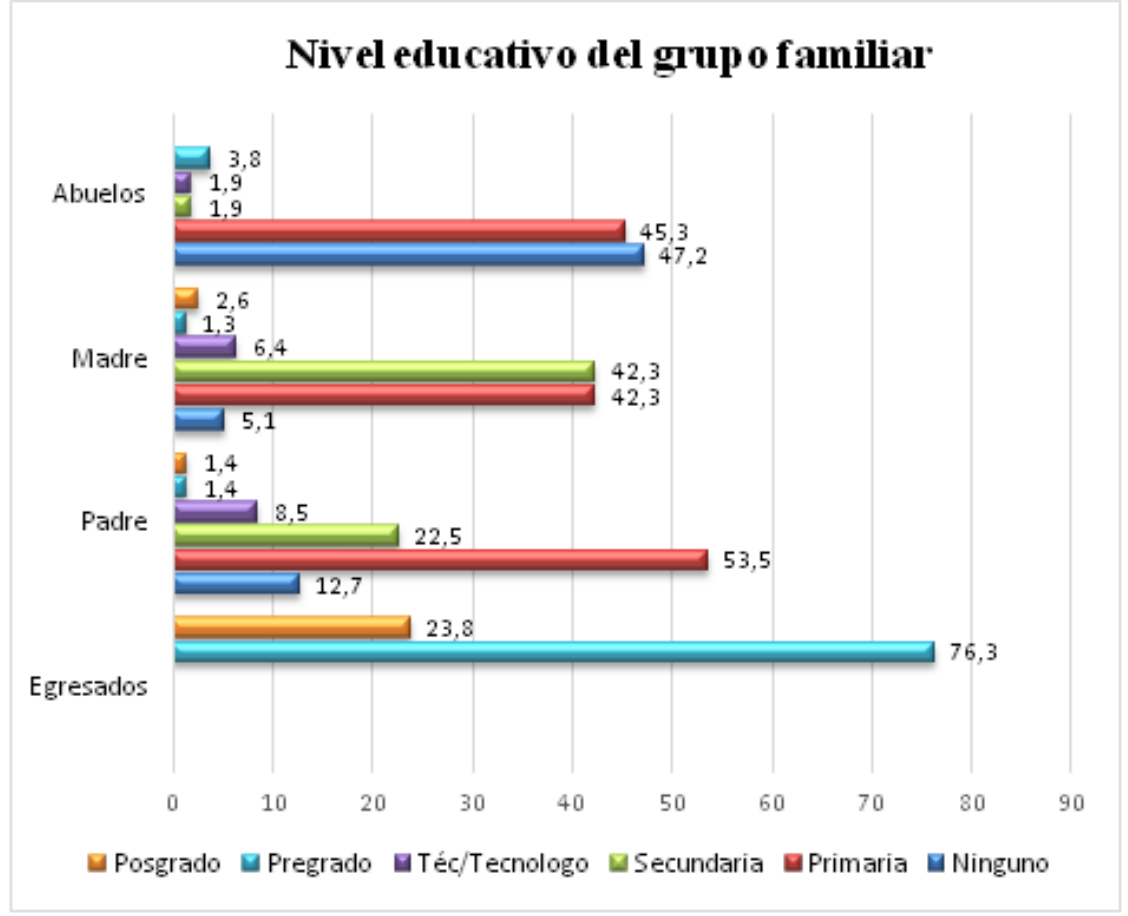

Fuente: Elaboración propia.

Los datos presentados permiten observar una baja, a veces nula, participación familiar en los últimos escalafones del logro educativo, especialmente entre la educación media escolar y la educación superior y posgraduada. Aunque se analiza un comportamiento creciente entre generaciones, en términos de logro educativo, se trata de familias que en su mayoría no accedieron a la universidad y a las prácticas y conocimientos que ella implica. No obstante, es relevante que las expectativas que poseen los padres, y otros grupos, en el establecimiento de discursos sobre el acceso a la educación superior constituyen una clave para entender la conducta y el obrar de estos jóvenes. Así lo muestra que el $80 \%$ de los padres les manifestaron a sus hijos e hijas algún tipo de expectativa e interés asociado a la necesidad de que accedieran a la educación superior; las cuales tenían que ver con la realización personal, el progreso económico, el progreso intelectual y la tradición familiar, solo el $20 \%$ de los padres no manifestó expectativa alguna. La media de la realización personal es de 4,78, en una escala de 1 a 5 , con una desviación estándar de 0,573 , y la media del progreso intelectual 4,71 con una desviación estándar de 0,578. 
En consecuencia, con las expectativas de estudio, el 96,3\% de estos jóvenes recibió apoyo cognitivo, económico o emocional por parte de sus familiares, los cuales incluyen abuelos, tíos, hermano y pareja. Esto explica que aunque la educación superior y posgraduada no se presente como una tradición relevante en la familia, se trata de grupos que impulsan en sus hijos una serie de valores, discursos y motivaciones asociados a la educación superior.

Aunado a los resultados anteriores, es de resaltar que en el éxito del proceso formativo no solo basta con acceder a los centros de formación, sino que es necesario cumplir con ciertos indicadores de rendimiento escolar, dado que es el medio concreto por el cual la experiencia intelectual y cognitiva del proceso de aprendizaje queda plasmado. Al respecto, se encontró que el rendimiento escolar de estos jóvenes fue sobresaliente, bajo el sistema colombiano de calificaciones de 5 puntos, la media de promedios acumulados al graduarse es de 4,26 , con una moda de 4,20 y una desviación estándar de 0,24. En la percepción propia, el 95\% calificó su rendimiento académico como excelente o bueno. Adicionalmente, es importante destacar que el 61,3\% de estos jóvenes trabajó durante la mayor parte del pregrado, el 16,3\% eventualmente durante algunos semestres o los fines de semana, tan solo el 22,4\% no trabajó durante el pregrado, lo que revela, en gran parte, que la formación educativa estuvo en la mayoría de estos jóvenes rodeada de múltiples retos, ya sean factores internos y externos, que hace del logro educativo alcanzado un reflejo de las capacidades de asumir responsabilidades, pues esta decisión de acceder a determinados espacios educativos demanda un esfuerzo físico, económico, cognitivo y ético.

\subsection{MOTIVACIÓN PROFESIONAL}

Salir al mercado laboral es una de las mayores dificultades de los jóvenes profesionales en la actualidad; la demanda de un cargo estable y un alto reconocimiento salarial son recursos que cada vez se disputan con mayor afán en los circuitos económicos y empresariales. Los jóvenes aquí abordados lograron, con todos los desafíos posibles, «sacar adelante» sus carreras; en donde sus motivaciones se vieron puestas a prueba, y en el devenir de sus capacidades de agencia, las acciones y toma de decisiones, les posibilitaron terminar el proceso del pregrado de una manera eficaz.

La etapa de ejercer la profesión no solo implica el proceso de incursión al mundo laboral, también la capacidad que tienen los sujetos de adaptarse a los 
diversos contextos espacio-temporales en los que participan. En lo concerniente a la vida laboral de la población estudiada, se observa que después de egresar, más de la mitad -el 57,5\% - logró emplearse en un lapso de dos meses aproximadamente; el resto lo hizo en un lapso de cinco o más meses. En la actualidad, el $86,25 \%$ de la población considera que ejerce la carrera en la que se formaron, la mayoría de estos jóvenes (84\%) trabaja en el sector académico y de servicios; el 95,7\% afirma que lo hace en condiciones formales, entendiendo por esto a la forma de contratación legalmente sancionada por el Estado (asalariados e independientes); el 5,8\% expresó ejercer en condiciones no formalizadas, realizando labores sin contrato y de forma voluntaria. El 13,75\% de los casos dice no ejercer.

\section{GRÁFICO 2. CONDICIONES LABORALES DE LOS JÓVENES QUE EJERCEN LA} PROFESIÓN

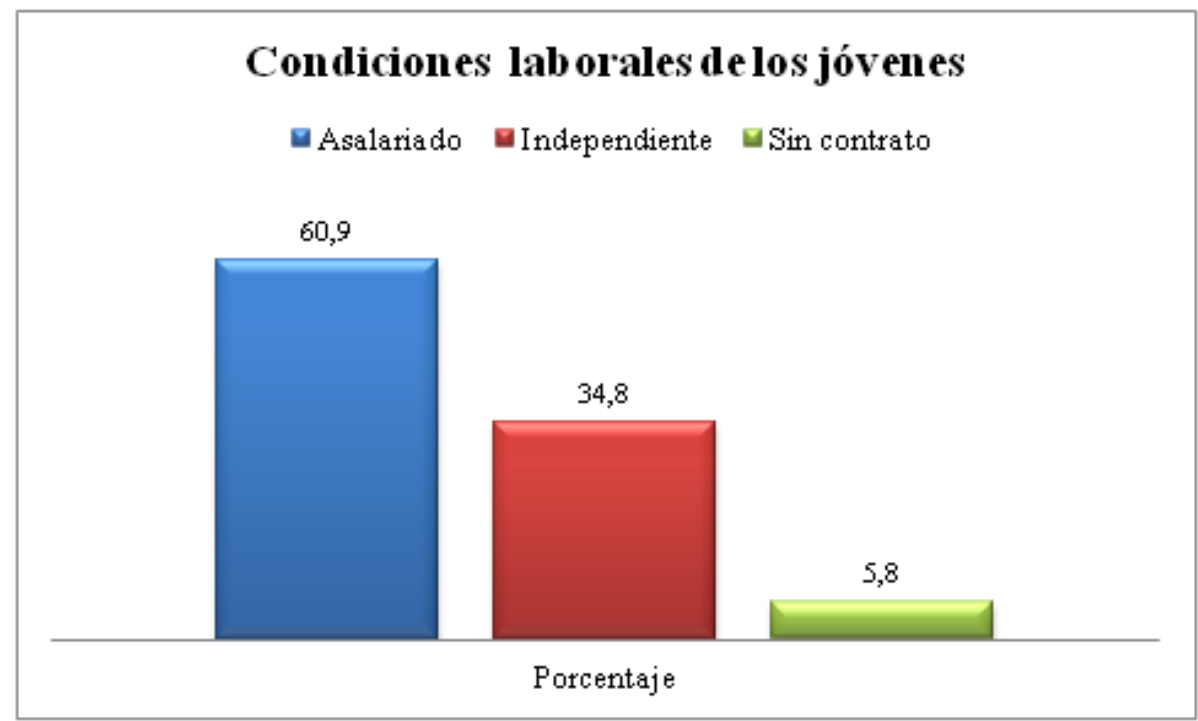

Fuente: Elaboración propia.

Esto significa que la mayoría logró adquirir trabajos y ocupaciones acordes a la formación recibida, que, además, se trata de un grupo que se mueve entre distintas formas de contratación laboral, que van desde el trabajo independiente, por prestación de servicios, trabajo asalariado y voluntariado; lo que resulta bastante relevante en términos de logro profesional, al indicar el grado 
de utilidad para estos jóvenes de una formación académica que logra cohesionarse en las dinámicas laborales de la economía intelectual o calificada.

Una de las características más relevantes analizadas es conseguir empleo, puesto que plasma la relación costo/beneficio; sin embargo, dadas las cualidades del mercado laboral para jóvenes, los primeros empleos suelen ser trabajos con ciertas precariedades salariales y de prestaciones sociales. En lo tocante a su estado de ingresos, se observa que poco más de la mitad, esto es, un 52,5\% de los casos, gana entre uno y menos de tres salarios mínimos legales vigentes ${ }^{5}$, ingresos que se asemejan a los ingresos totales del grupo familiar al momento de ingresar a la universidad; teniendo que el $28,7 \%$ gana entre tres y menos de cinco salarios mínimos. Mostrando así, que la diferencia generada en términos de crecimiento económico no es muy significativa, dato que se corresponde con la satisfacción salarial de los jóvenes, pues más de la mitad afirman no estar del todo satisfechos con sus ingresos. Según Carvajal Restrepo (2018), en Colombia un profesional con pregrado debería ganar sin experiencia en el área de formación y con contrato a término fijo o indefinido un aproximado de 694 dólares $^{6}$ mensuales, y en el mismo caso, pero con un contrato por prestación de servicios, 1,027 dólares. Este bajo reconocimiento económico se debe a razones que destacan los jóvenes, como la poca oferta de empleos y exigencia de una amplia experiencia laboral.

A pesar de la baja satisfacción salarial de los jóvenes, que denota un entorno desafiante en lo económico, se evidencia en contraste que el aspecto que más los motivó a ser profesionales fue la realización personal, respecto de lo cual se encontró que el $86,3 \%$ de estos jóvenes considera que en el ámbito personal son profesionales casi o totalmente exitosos; denotando así la consolidación de las motivaciones en logros, sin importar los entornos desafiantes que pusieron a prueba sus capacidades de reflexión y ejecución para conseguir concretar metas de su proyecto de vida a través de la educación.

${ }^{5}$ El salario mínimo mensual en Colombia es de 877.803 pesos para el año 2020, que corresponde a 236,4 dólares aproximadamente.

${ }^{6}$ Se hace la conversión de pesos colombianos a dólares estadounidenses para una mejor comprensión en regiones fuera de Colombia, aplicando el valor promedio del dólar de 2.952 pesos colombianos para el año de la publicación citada. 


\section{REFLEXIONES FINALES}

En esta investigación se observa que la motivación de los jóvenes abordados, y de sus familiares, es un aspecto que explica la conducta y el logro educativo y profesional. Frente a la trayectoria escolar de sus estructuras familiares, la mayoría de ellos son pioneros en la formación superior, incluso presentándose casos en los que ningún integrante del grupo familiar accedió a la educación formal. Ello permite reflexionar el impacto que juegan estos jóvenes, puesto que es probable que influyan en los cursos de vida de las nuevas generaciones, las que podrán ser herederas de un capital cultural más consolidado (Mata Zúñiga, 2012). Los resultados muestran que el grupo analizado de jóvenes adquirió un capital educativo y profesional importante frente a las cadenas de reproducción social de sus generaciones pasadas. No obstante, los familiares jugaron un papel importante en la construcción de exigencias y demandas a sus hijos, pues introdujeron discursos y expectativas sobre la vida educativa, que se convirtieron en logro a partir de la voluntad y el empeño de estos jóvenes. Una vez ingresaron a la vida académica, el rendimiento escolar, tanto en su percepción como en las calificaciones, fue positivo y alto, lo que mostró su capacidad para emprender acciones que fueran más allá del cumplimiento de requisitos para obtener el título. Al finalizar el proceso de formación, la mayoría logró ejercer su carrera al enfrentarse con las dinámicas propias del mercado laboral, lo que vista la capacidad de estos para movilizarse entre distintas responsabilidades y demandas sociales. Tanto la motivación como el logro son factores que expresan la capacidad que tuvieron para transformar sus entornos inmediatos.

Para algunos estudios sobre la desigualdad, existe un supuesto en las ciencias sociales que asegura que los jóvenes con recursos escasos tendrán mayor riesgo de desertar o de obtener un bajo rendimiento académico (Rodríguez Urrego, 2019; Izquierdo Cázares y Mestanza Páez, 2017). Muchas de estas ideas explican que, por ejemplo, en contextos como el colombiano, alrededor del $37 \%$ de los estudiantes que comienza un programa universitario abandona el proceso formativo, el $36 \%$ desertan tan solo en el primer año de la formación (Pérez Martínez, 2017; Tarabini y Curran, 2015). Un estudiante que llega con diferentes carencias académicas y de redes intelectuales, tiene un costo de oportunidad mucho mayor para acceder a la cultura de la educación formal, lo que puede motivar, consecuentemente, su deserción. Sin embargo, estas carencias, que pueden ser de diversa índole, deben ser comprendidas de forma dual, constriñen y niegan diversas oportunidades de movilidad social a la vez que habilitan otros 
aspectos de la realidad social. Tal como se observa, se trata de un comportamiento bajo el cual es posible relacionar la falta de educación de los familiares con una motivación duradera de los jóvenes para lograr superar y alcanzar aquello que sus ascendientes no lograron.

Los estudios de la desigualdad aseguran que el origen social posee un fuerte impacto en el futuro de los agentes humanos, y aunque esto es cierto sobre todo en la esfera salarial, no se puede asegurar que estas fuerzas sociales «empujen» a nadie a hacer cosa alguna si no ha sido ya arrastrado previamente (Giddens, 2011). Vale decir, que la familia, el otro(s) colectivo, en vez de generar un impacto negativo, se comportó como agente de socialización, lo cual fue clave dado que jugó un rol central en la transmisión de valores y expectativas que desembocaron en motivaciones y oportunidades educativas reales (Reyes Alamilla y Hernández Romero, 2019; Rodríguez, 2016; Tarabini y Curran, 2015: 21 ), siendo relevante establecer que estos jóvenes se convirtieron en un punto de referencia y liderazgo dentro de sus grupos familiares. En efecto, se puede realizar una interpretación optimista sobre las trayectorias juveniles como parte de la estructura (Dávila León y Ghiardo Soto, 2018), y más a partir de los resultados obtenidos, y en concordancia con algunos estudios que aseguran que las decisiones educativas de los jóvenes van más allá de su contexto familiar. Las principales consecuencias en el futuro de estos jóvenes se relacionan directamente con el nivel de satisfacción personal y profesional.

Es evidente que la edad vivida de una persona tiene un gran impacto sobre el bienestar subjetivo. Las demandas y exigencias de logro educativo y laboral no se materializan sin un sujeto que tenga la capacidad de superar aquellos aspectos que escapan a la voluntad del sujeto. Es necesario comprender que los resultados de este estudio ponen de manifiesto la existencia de una capacidad de agencia por parte de los jóvenes abordados, tal como lo proponen otros autores (Ruiz González et al., 2018); capacidad que debe ser entendida como el resultado de la forma de vivencia del conflicto entre los jóvenes donde sus relaciones salen fortalecidas al afrontar, de manera constructiva y recursiva, las situaciones de desigualdad y de tensión (Arias Cardona y Arias Gómez, 2018: 65). En este sentido, la educación superior es un medio, con un valor social intrínseco, que les permitió a estos jóvenes moverse en una estructura de desigualdades que funge como frenadores de los sueños, expectativas, intereses y motivaciones de los agentes.

A pesar de que muchos son los jóvenes que realmente sí desertan y no consolidan un proyecto de vida en la educación, no se puede asegurar, bajo algún 
tipo de normativa cultural, que la edad determine de forma unidireccional - es decir, sea causa absoluta - la capacidad social, emocional y cognitiva de los seres humanos. Mucho se ha debatido con respecto a la edad en que se debe egresar de los colegios e institutos de educación media-bachillerato e ingresar a las instituciones de educación superior, si existen edades muy tempranas o si se trata de un comportamiento «natural» de las sociedades contemporáneas. Este estudio permite concluir que, a la hora de decidir el futuro, nunca se es muy joven o muy adulto, pues lo verdaderamente importante recae en las variables y condiciones personales, motivacionales y reflexivas del sujeto que opta por transformar su vida. En este sentido, los jóvenes tienen una capacidad de agencia que se debe, en gran parte, a una capacidad personal de apropiación y transformación de su realidad de vida, donde la educación impulsa procesos de movilidad social intergeneracional para el logro educativo. Estos datos aportan apoyo empírico, sumándose a otras investigaciones (Ruiz González et al., 2018), sobre la importancia de desarrollar programas que refuercen la autoestima de estudiantes universitarios, pues simultáneamente se incrementarían los niveles de satisfacción global con la vida.

Aunque es cierto que en la actualidad la expansión de la educación superior y el aumento del número de matriculados en muchos países de Latinoamérica son un hecho, todavía más para las generaciones actuales, también es real que: en primer lugar, en territorios como en los que se formó la población de jóvenes aquí abordada, el acceso a la educación superior pública es casi inexistente, y las posibilidades de financiar una educación privada para estos es por lo menos inconmensurable. En segundo lugar, es posible hablar de una movilidad educativa intergeneracional, punto que agrega una acción social reflexiva que les demandó implicaciones lógicas en el futuro a estos individuos, que posee un registro personal e intelectual en la conciencia de los agentes implicados, incluyendo sus grupos familiares y diversos actores que intervienen de oficio. En consecuencia, puede decirse que no existe una edad para decidir por la educación, en razón que la edad no es un determinante universal de la agencia humana, debido a esto, todos los seres individuales, aun en las situaciones más complejas y amenazadoras, realizan esfuerzos para solventar dicha carga de dependencia (Giddens, 2011). En la actualidad, los estudios de egresadas y egresados contribuyen a valorar el efecto de la educación superior, proporcionando datos para generar mayores procesos de desarrollo social y económico. Es de sugerir que actores gubernamentales, locales e institucionales tengan intereses en conocer la capacidad que poseen los jóvenes para el cambio, 
frenando con ellos la alta segregación laboral, la falta de oportunidades sociales y el poco valor que se le otorga a su voz.

RECIBIDO: 4 DE DICIEMBRE DE 2020

ACEPTADO: 20 DE ENERO DE 2021

\section{BIBLIOGRAFÍA}

Angelucci, L. T., CAÑoto, Y. y Hernández, M. J. (2017). Influencia del estilo de vida, el sexo, la edad y el IMC sobre la salud física y psicológica en jóvenes universitarios. Avances en Psicología Latinoamericana, 35(3), 531-546. Recuperado de: http://www.scielo.org.co/pdf/apl/v35n3/17944724-apl-35-03-00531.pdf

ARANDA, B. Y. (2016). Las organizaciones de la sociedad civil de asistencia. Una descripción cualitativa, desde la teoría de estructuración de Anthony Giddens. Recuperado de: http://ru.iiec.unam.mx/3271/1/218-Aranda.pdf

Arias-Cardona, A. M. y Soto, J. A. (2019). Encuentros con la diferencia: la pluralidad en las experiencias de jóvenes universitarios. Psicología desde el Caribe, 36(1), 45-60. Recuperado de: http://www.scielo.org.co/pdf/psdc/v36n1/2011-7485-psdc-36-01-45.pdf

Arias-Cardona, A. M. y Arias-Gómez, M. (2018). Conflicto y educación superior: narrativas y vivencias de jóvenes universitarios estudiantes de ciencias sociales y humanas. Rev. CES Psico, 11(1), 56-68. Recuperado de:

https://www.redalyc.org/jatsRepo/4235/423557502006/html/index.html

BAR, A. R. (2010). La Metodología Cuantitativa y su uso en América Latina. Cinta de moebio, (37), 1-14. Recuperado de: https://dx.doi.org/10.4067/S0717-554X201000010000

Bialakowsky, A. (2017). La temporalidad y la contingencia en el «giro del sentido» propuesto por las perspectivas teóricas de Giddens, Bourdieu, Habermas y Luhmann. Sociológica (México), 32(91), 9-44. Recuperado de: $\quad$ http://www.scielo.org.mx/pdf/soc/v32n91/2007-8358-soc-32-9100009.pdf

. (2018). Vida cotidiana y reclasificaciones sociológicas según Giddens, Bourdieu, Habermas y Luhmann. Convergencia, 25(77), 125-147. Recuperado de: http://www.scielo.org.mx/pdf/conver/v25n77/24485799-conver-25-77-125.pdf 
BLANCO, Ó. A. (2007). La promoción del empleo juvenil y la capacitación para el trabajo en Colombia. Revista Latinoamericana de Derecho Social, (5), 47-63. Recuperado

de: https://www.redalyc.org/pdf/4296/429640260003.pdf

Cartagena, K. (2005). Movilidad intergeneracional en Colombia. Ensayos sobre política económica (ESPE), (51), 208-265. Recuperado de: https://www.banrep.gov.co/sites/default/files/publicaciones/archivos/esp e 051-4.pdf

Carvajal, E. (2018). Esto es lo que debería ganar según su formación y experiencia. Periódico el Colombiano. Recuperado de: https://www.elcolombiano.com/negocios/economia/escala-salarial-encolombia-XG8484123

CAstillo, D. B. (2019). La causalidad histórica: la aporía entre el agente y la estructura en algunas corrientes historiográficas del siglo XX. Algunos elementos para su discusión actual. Letras Históricas, (21), 215-243. Recuperado de: http://www.scielo.org.mx/scielo.php?script=sci arttext\&pid=S2448$\underline{83722019000200215 \& \operatorname{lng}=\mathrm{es} \& \ln \mathrm{ln}=\mathrm{es}}$

Chávarro, L. A. (2018). Riesgo e incertidumbre como características de la sociedad actual: ideas, percepciones y representaciones. Revista Reflexiones, 97(1), 65-75. Recuperado de: https://www.scielo.sa.cr/pdf/reflexiones/v97n1/1659-2859-reflexiones97-01-65.pdf

CoHEN, S. (2017). Prejuicios y mitos sobre el adolescente que crece. Clarin.com. Recuperado de: https://www.clarin.com/revista-enie/ideas/prejuiciosmitos-adolescente-crece_0_Skmt_Nkqb.html

Córdova, M., Rosales, M. D. P., Caballero, R. y Rosales, C. J. (2007). Ideación Suicida en Jóvenes Universitarios: su Asociación con Diversos Aspectos Psicosociodemográficos. Psicología, 15(2), 17-21. Recuperado de: https://www.redalyc.org/pdf/1339/133915933003.pdf

Corominas, J. (2016). Breve introducción a la sociología del cuerpo de Anthony Giddens. Perifèria. Cristianisme, postmodernitat, globalització, 3(3), 121-133. Recuperado de: https://www.raco.cat/index.php/PeriferiaCPG/article/viewFile/332464/4 23222

CruZ, J. D. J. (2017). El concepto de experiencia en Victor W. Turner, EP Thompson y Anthony Giddens: Un diálogo entre antropología social, historia y sociología. Sociología Histórica, (7), 345-375. Recuperado de: https://dialnet.unirioja.es/descarga/articulo/6341751.pdf 
DÁVILA, O. y GHIARDO, F. (2018). Trayectorias sociales como enfoque para analizar juventudes. Última Década, 26(50), 23-39. Recuperado de: https:/ultimadecada.uchile.cl/index.php/UD/article/view/53843/56597

Denegri, M., Sepúlveda, J., Etchebarne, S., GonzÁlez, Y., Gempe, R. y Del VALLE, C. (2009). Construcción de representaciones acerca de pobreza y movilidad social: Un estudio evolutivo en estudiantes chilenos. Paideia, (46), 159-176. Recuperado de: https://revistas.udec.cl/index.php/paideia/article/view/1832

EspinosA, A. M. (2017). Estadística para las Ciencias Sociales y Humanas I. Bogotá: Fundación Universitaria del Área Andina. Disponible en: https://digitk.areandina.edu.co/bitstream/handle/areandina/1218/Estad $\%$ C3\%ADstica\%20para\%20las\%20Ciencias\%20Sociales\%20y\%20Huma nas $\% 20$ I.pdf?sequence $=1 \&$ isAllowed $=y$

FILARDO, V. (2018). Juventud, juventudes, jóvenes: esas palabras. Última Década, 26(50), 109-123. Recuperado de: https://scielo.conicyt.cl/pdf/udecada/v26n50/0718-2236-udecada-26-5000109.pdf

GARCÍA, C. (2017). Movilidad intergeneracional educativa en zonas urbanas y rurales de Colombia: ¿Igualdad de oportunidades? Sophia, 13(1), 27-33. Recuperado de: https://doi.org/10.18634/sophiaj.13v.1i.366

GidDENS, A. (1992). Sociología (2a ed.). Madrid: Alianza Editorial. . (1997). Modernidad e identidad del yo: el yo y la sociedad en la época contemporánea. Barcelona: Península.

. (2000a). La estructura de clases de las sociedades avanzadas. Madrid: Alianza Editorial.

. (2000b). Sociología (3a ed.). Barcelona: Alianza Editorial.

- (2011). La constitución de la sociedad: bases para la teoría de la estructuración (2a ed.). Buenos Aires: Amorrortu.

. (2012). Las nuevas reglas del método sociológico. Crítica positiva de las sociologías comprensivas. Madrid: Amorrortu.

. (2018). Perfiles y críticas en teoría social. Buenos Aires: Prometeo.

Giddens, A. y Muro, J. B. (1979). La estructura de clases en las sociedades avanzadas ( $\left.1^{\mathrm{a}} \mathrm{ed}.\right)$. Madrid: Alianza Editorial.

Giddens, A. y Turner, J. (1990). La teoría social hoy (1a ed.). Barcelona: Alianza Editorial.

Hernández, R., Fernández, C. y Baptista, M. D. P. (2014). Metodología de la investigación ( $6^{\mathrm{a}}$ ed.). Ciudad de México: McGraw Hill. Recuperado de: 
content/uploads/2017/08/metodologia-de-la-investigacion-sextaedicion.compressed.pdf

IzQuierdo, G. y MestanzA, R. C. (2017). Retos de la educación ante la deserción escolar universitaria. Revisión sistemática. Revista Científica Retos de la Ciencia, 1(2), 15-21. Recuperado de: https://core.ac.uk/download/pdf/229098253.pdf

Jaramillo, C. A., Arias, A. M., Arias, M. R., Restrepo, F. I. y Ruiz, D. A. (2012). Relaciones sociales entre jóvenes universitarios: una mirada desde las lógicas subjetivas. Envigado, Antioquia: Institución Universitaria de Envigado-IUE y Fundación Universitaria Luis Amigó. Recuperado de: https://www.iue.edu.co/relaciones-sociales-entre$\mathrm{j} \% \mathrm{C} 3 \% \mathrm{~B} 3$ venes-universitarios-una-mirada-desde-las-1\%C3\%B3gicassubjetivas.html

Kerbo, H. R. (2003). Estratificación Social y Desigualdad. El conflicto de clase en perspectiva histórica, comparada y global. Madrid: McGraw Hill/Interamericana de España, S.A.U.

MAtA, L. A. (2012). Entre los estudios y el empleo. Estrategias de inserción social en egresados de la Universidad Nacional Autónoma de México. Universidades, 29-42. Recuperado de: http://udualerreu.org/index.php/universidades/article/view/216/224

Moo, E. G. y LóPEz, R. (2019). Becarios de clase media en una universidad privada del sureste de México. Formas de convivencia, perspectivas académicas y de futuro laboral. Península, 14(1), 9-28. Recuperado de: http://www.scielo.org.mx/pdf/peni/v14n1/1870-5766-peni-14-01-9.pdf

Otzen, T. y Manterola, C. (2017). Técnicas de Muestreo sobre una Población a Estudio. International Journal of Morphology, 35(1), 227-232. Recuperado de: https://scielo.conicyt.cl/pdf/ijmorphol/v35n1/art37.pdf

Pavez Soto, I. y Sepúlveda Kattan, N. (2019). Concepto de agencia en los estudios de infancia. Una revisión teórica. Sociedad e Infancias, 3, 193210. Recuperado de: https://doi.org/10.5209/soci.63243

PÉrez MartíneZ, A. (2017). En Colombia el 40\% de los jóvenes no terminan la educación media. Revista Dinero. Recuperado de: https://www.dinero.com/opinion/columnistas/articulo/en-colombia-losjovenes-no-terminan-la-educacion-media-angel-perez/242654

Reyes Alamilla, O. I. y Hernández Romero, G. (2019). Identificación y práctica de valores en la formación universitaria. Recuperado de: http://scielo.sld.cu/pdf/rces/v38n2/0257-4314-rces-38-02-e17.pdf

RODRÍGUEZ, M. (2019). La investigación sobre deserción universitaria en Colombia 2006-2016. Tendencias y resultados. Pedagogía y Saberes, 51, 
49-66. Recuperado de: https://doi.org/10.17227/pys.num51-8664

RODRÍGUEZ, S. A. (2016). Logros educativos en el nivel de instrucción superior y movilidad educacional intergeneracional en Argentina. Sociológica (México), 31(88), 167-200. Recuperado de: https://www.redalyc.org/articulo.oa?id=305045555006

Ruiz-GonzÁlez, P., Medina-Mesa, Y., Zayas, A. y Gómez-Molinero, R. (2018). Relación entre la autoestima y la satisfacción con la vida en una muestra de estudiantes universitarios. International Journal of Developmental and Educational Psychology, 2(1), 67-76. Recuperado de:

http://www.infad.eu/RevistaINFAD/OJS/index.php/IJODAEP/article/vi $\underline{\text { ew/1170/1038 }}$

SAntos OrtegA, A. y MuÑoz-Rodríguez, D. (2017). La subjetivación del capital humano: la movilidad como inversión en los discursos de los jóvenes universitarios que han protagonizado la fuga de cerebros. Areas. Revista Internacional de Ciencias Sociales, (36), 127-139. Recuperado de: https://revistas.um.es/areas/article/view/308111

SÉMBLER, C. (2006). Estratificación social y clases sociales. Una revisión analítica de los sectores medios. Santiago de Chile: CEPAL. Recuperado de:

https://repositorio.cepal.org/bitstream/handle/11362/6130/S0600897 es. pdf;

TARABINI, A. y CURRAN, M. (2015). El efecto de la clase social en las decisiones educativas: un análisis de las oportunidades, creencias y deseos educativos de los jóvenes. Revista de Investigación en Educación, 13(1), 7-26. Recuperado de: http://reined.webs.uvigo.es/index.php/reined/article/view/239/273

TismashefF, N. (1961). Teoría sociológica. Ciudad de México: Fondo de Cultura Económica.

UniVERSIDAD DE ANTIOQUiA. (14 de julio de 1998). Acuerdo Académico 0133, por medio del cual se definen la misión, los principios, los objetivos y las políticas de Regionalización de la Universidad.

Vega, C., Gómez, C. y Monteros, S. (2017). Jóvenes ecuatorianos, movilidad $\mathrm{y}$ educación superior en España y Ecuador. Trayectorias educativas desiguales en tiempos de crisis. Athenea Digital. Revista de pensamiento e investigación social, 17(3), 173-198. Recuperado de: https://doi.org/10.5565/rev/athenea.1815

WeBer, M. (2002). Economía y sociedad: esbozo de sociología comprensiva. Ciudad de México: Fondo de Cultura Económica.

Westendarp, P. e IGLESIAS, L. G. (2019). Jóvenes Universitarios y Sociabilidad. 
Última Década, 27(51), 3-27. Recuperado de: https://ultimadecada.uchile.cl/index.php/UD/article/view/54291/60377

YASCHINE, I. (2015). ¿Alcanza la educación para salir de la pobreza? Análisis del proceso de estratificación ocupacional de jóvenes rurales en México. Revista mexicana de ciencias políticas y sociales, 60(223), 377-405. Recuperado de: http://www.scielo.org.mx/scielo.php?pid=S0185$\underline{19182015000100014 \& \text { script }=\text { sci abstract }}$ 